\title{
Hadron-Hadron Correlation and Interaction from Heavy-Ion Collisions
}

\author{
Akira Ohnishi* \\ Yukawa Institute for Theoretical Physics, Kyoto University, Kyoto 606-8502, Japan \\ E-mail: ohnishi@yukawa.kyoto-u.ac.jp
}

\begin{abstract}
We investigate the $\Lambda \Lambda, N \Omega$ and $K^{-} p$ momentum correlations in high-energy heavy-ion collisions and their relevance to the hadron-hadron interactions. For $R / a_{0}<0,\left|R / a_{0}\right| \ll 1$ and $R / a_{0}>1$, where $R$ and $a_{0}$ denote the source size and scattering length, the correlation functions at small relative momenta are enhanced, strongly enhanced and suppressed, respectively, by the interaction when the interaction range is short and the single channel treatment is justified. The recently observed $\Lambda \Lambda$ correlation function is found to be enhanced by the interaction from that by the quantum statistics with the feed-down effecs, provided that the pair purity is as large as the statistical model estimate. The scattering length of the $\Lambda \Lambda$ interaction is constrained to be $1 / a_{0}<-0.8 \mathrm{fm}^{-1}$ by the correlation data. For the $\Omega^{-} p$ correlation, we propose to introduce an "SL (small-to-large) ratio" of the correlation functions for different source sizes in order to evade the contamination by the Coulomb interaction. In the SL ratios, the above characteristic interaction dependence is found to be recovered. Then the SL ratio is useful to judge the sign and strength of the scattering length and consequently the existence of the $S=-3$ dibaryon state. The coupling effects of the $K^{-} p$ and $\bar{K}^{0} n$ channels are found to be important for the $K^{-} p$ correlation. The outgoing wave function in the $K^{-} p$ channel differs from the complex conjugate of that in the $K^{-} p$ scattering due to the coupled-channel effects. Then we may find peak and dip structures different from those in the $K^{-} p$ scattering cross section, and it would be possible to examine the interference of the $I=0$ and $I=1$ amplitudes.
\end{abstract}

The 26th International Nuclear Physics Conference

11-16 September, 2016

Adelaide, Australia

${ }^{*}$ Speaker. 


\section{Introduction}

Variety of hadrons are produced abundantly in high-energy heavy-ion collisions. Their yields, nuclear modification factors, flows and fluctuations tell us the properties of created hot and dense matter such as $(T, \mu)$, jet quenching, parton collectivity and the critical point. Heavy-ion collisions are also useful to study hadron physics. Medium effects and formation mechanisms of hadrons may be investigated via the invariant mass spectra and production yields [1]. Furthermore, it is possible to study interaction between hadrons by using the two-particle momentum correlation. The twoparticle correlation is generated by quantum statistics and final state interactions, and also affected by the size and lifetime of the emission source. Thus if the property of the source is known, we can access the hadron-hadron interaction. This aspect is extremely useful to explore interactions between short-lived particle pairs. In recent experiments, correlations have been measured for particle pairs such as $p \bar{p}, \bar{p} \bar{p}, p \Lambda, \bar{p} \Lambda(p \bar{\Lambda})$ and $\Lambda \Lambda$. It would be also possible to measure the correlations for pairs such as $\Omega^{-} p, K^{-} p$ and $\Xi^{-} p$. The interactions in these pairs can serve as crucial inputs to understand possible exotic hadrons and exotic matter such as dibaryons and hadronic molecules.

In this proceedings, we discuss the $\Lambda \Lambda$ [2], $\Omega^{-} p$ [4] and $K^{-} p$ [3] interactions by using correlations measured or to be measured in heavy-ion collisions based on our recent works.

\section{Hadron-hadron correlation function in heavy-ion collisions and its interaction dependence}

The two-particle momentum correlation function $C(\boldsymbol{q})$ is defined as the ratio of the twoparticle production probability to the product of single particle spectra. When the emission source is chaotic, $C(\boldsymbol{q})$ is obtained by using so-called the Koonin-Pratt (KP) formula [5, 6],

$$
C(\boldsymbol{q})=\frac{E_{1} E_{2} d N_{12} / d \boldsymbol{p}_{1} d \boldsymbol{p}_{2}}{\left(E_{1} d N_{1} / d \boldsymbol{p}_{1}\right)\left(E_{2} d N_{2} / d \boldsymbol{p}_{2}\right)} \simeq \int d \boldsymbol{r} S_{12}(\boldsymbol{r})\left|\varphi^{(-)}(\boldsymbol{r}, \boldsymbol{q})\right|^{2},
$$

where $\boldsymbol{q}=\left(E_{2} \boldsymbol{p}_{1}-E_{1} \boldsymbol{p}_{2}\right) /\left(E_{1}+E_{2}\right)$ is the relative momentum. When the emission time difference is small, $\varphi^{(-)}(\boldsymbol{r}, \boldsymbol{q})$ can be identified as the relative wave function with the outgoing momentum $\boldsymbol{q}$. The relative coordinate source function $S_{12}(\boldsymbol{r})$ is obtained from the single particle source functions.

When the single particle sources are spherical Gaussians and the particles in the pair are emitted simultaneously, $S_{i} \propto \delta\left(t_{i}-t_{0}\right) \exp \left(-\boldsymbol{x}_{i}^{2} / 2 R_{i}^{2}\right), S_{12}$ also becomes a Gaussian, $S_{12}(\boldsymbol{r})=$ $\exp \left(-\boldsymbol{r}^{2} / 4 R^{2}\right) /\left(4 \pi R^{2}\right)^{3 / 2}\left(R=\sqrt{\left(R_{1}^{2}+R_{2}^{2}\right) / 2}\right)$. In this case, the correlation function for the identical spin-half chargeless baryon pair is obtained as

$$
C_{B B}(q)=1-\frac{1}{2} e^{-4 q^{2} R^{2}}+\frac{1}{2} \Delta C(q), \quad \Delta C(q)=\int d \boldsymbol{r} S_{12}(\boldsymbol{r})\left[\left|\chi^{(-)}(r)\right|^{2}-\left(j_{0}(q r)\right)^{2}\right],
$$

where $\chi^{(-)}$denotes the wave function in the $s$-wave, and we assume that other partial waves are not modified. When the source size is sufficiently large compared with the interaction range, it becomes reasonable to replace the relative wave function with its asymptotic one, $\chi^{(-)}(r) \rightarrow \chi_{\text {asy }}(r)=$ $e^{-i \delta} \sin (q r+\delta) / q r=\mathscr{S}^{-1}\left(\sin q r / q r+f(q) e^{i q r} / r\right)$. By using $\chi_{\text {asy }}$ and the effective range correction, one arrives at the Lednicky-Lyuboshits (LL) formula [6],

$$
\Delta C^{\mathrm{LL}}(q)=\frac{1}{|\mathscr{S}|^{2}}\left[\frac{|f(q)|^{2}}{2 R^{2}} F_{3}\left(\frac{r_{\mathrm{eff}}}{R}\right)+\frac{2 \operatorname{Re} f(q)}{\sqrt{\pi} R} F_{1}(x)-\frac{\operatorname{Im} f(q)}{R} F_{2}(x)\right]+\frac{1-|\mathscr{S}|^{2}}{|\mathscr{S}|^{2}} \frac{F_{2}(x)}{x},
$$


where $x=2 q R, F_{1}(x)=\int_{0}^{x} d t e^{t^{2}-x^{2}} / x, F_{2}(x)=\left(1-e^{-x^{2}}\right) / x$ and $F_{3}\left(r_{\mathrm{eff}} / R\right)=1-r_{\mathrm{eff}} / 2 \sqrt{\pi} R$. The correlation function is given as a function of the scattering amplitude $f(q)$, which is described well by the scattering length $a_{0}$ and the effective range $r_{\text {eff }}$ at low energy, $f(q)=(q \cot \delta+i q)^{-1}$, $q \cot \delta=-1 / a_{0}+r_{\mathrm{eff}} q^{2} / 2+\mathscr{O}\left(q^{4}\right)$.
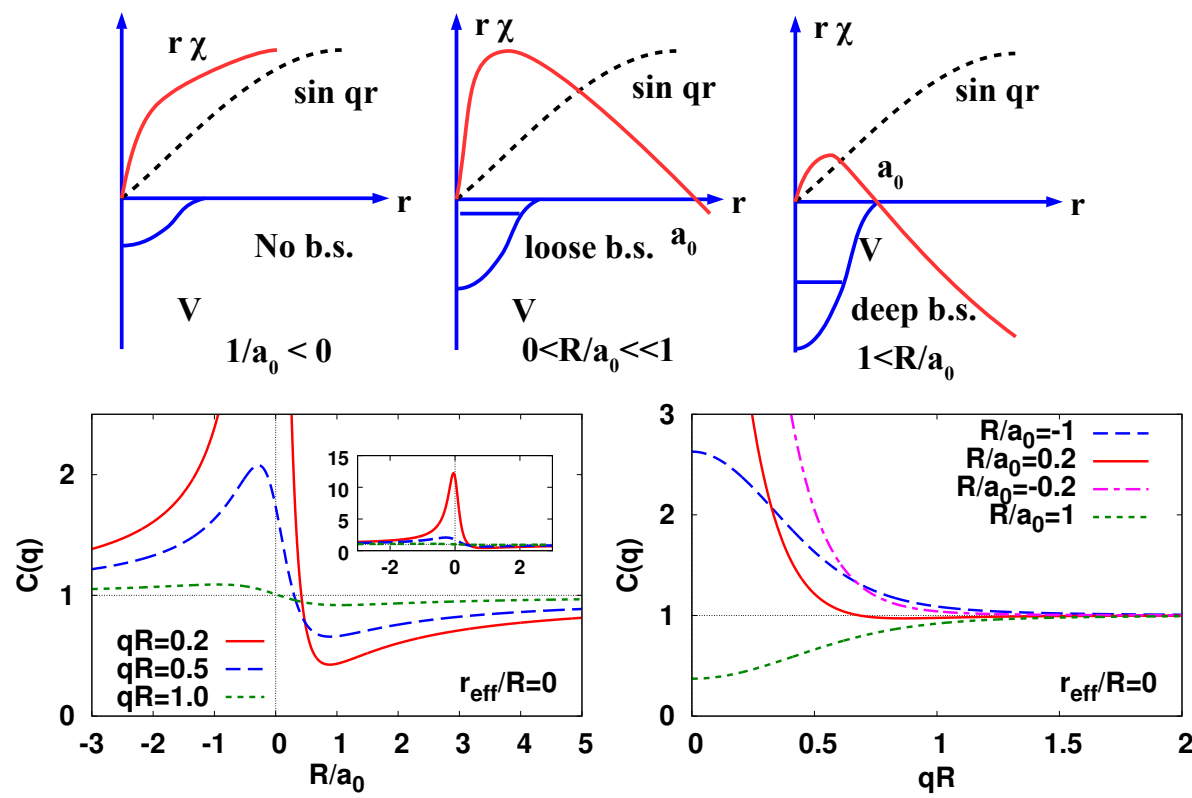

Figure 1: Upper panels: Schematic behavior of the relative wave functions for $R / a_{0}<0,0<R / a_{0} \ll 1$, and $R / a_{0}>1$. Lower panels: Correlation function in the LL model [6] as a function of $q R$ and $R / a_{0}$ in the case of zero effective range, $r_{\text {eff }} / R=0$.

In Fig. 1, we schematically show the wave functions for several values of $R / a_{0}$ and the interaction dependence of the correlation function. The asymptotic wave function $(r \chi)$ becomes a linear function of $r$ in the low energy limit, $r \chi_{\text {asy }}=e^{-i \delta} \sin \left(q r-a_{0} q\right) / q \rightarrow r-a_{0}(q \rightarrow 0)$, where we adopt the "nuclear physics" convention for the scattering length, $\delta \simeq-a_{0} q$ at low energy. The wave function is enhanced with $a_{0}<0$ and the correlation function is generally enhanced from unity. For $a_{0}>0$, the wave function has a node at $r \simeq a_{0}$. The node suppresses $|\chi(r)|^{2}$ at $r \lesssim R$ for a small scattering length, $a_{0} \lesssim R\left(R / a_{0} \gtrsim 1\right)$, while the wave function is enhanced for a large scattering length, $a_{0} \gg R\left(R / a_{0} \ll 1\right)$ [4]. As shown in the lower panels of Fig. 1, the correlation functions between non-identical particles in the LL model, $C(q)=1+\Delta C^{\mathrm{LL}}(q)$, demonstrate the above described interaction dependence. If these features survive other effects such as the Coulomb potential, quantum statistics, channel coupling and absorption, the correlation function data can provide information on the scattering length and hopefully other interaction parameters.

\section{Hadron-hadron interaction from correlation}

\section{1 $\Lambda \Lambda$ interaction}

The $H$ dibaryon (uuddss, $S=-2$ ) was first predicted to be a deep bound state below the $\Lambda \Lambda$ 
threshold [7]. Recent lattice QCD and ab initio few-body calculations also predict a bound $H$ in the SU(3) limit and/or at heavier quark masses [8]. While the experimental data do not show any signals of bound $H[9,10]$, the pole could evolve into a resonance near the $\Xi N$ threshold at physical quark masses [11], as suggested by the bump structure observed in the $\left(K^{-}, K^{+} \Lambda \Lambda\right)$ reaction [12]. Since the correlation function is sensitive to the scatering length, the $\Lambda \Lambda$ correlation provides important information on the pole position if $H$ exists around the $\Lambda \Lambda$ threshold [13].
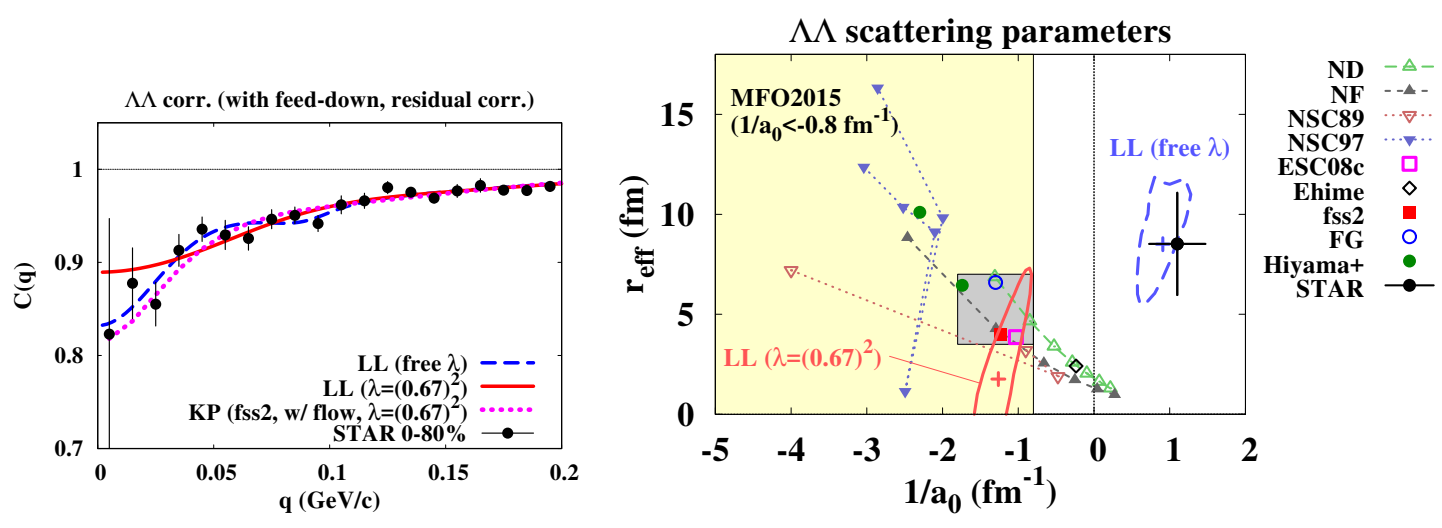

Figure 2: Left: $\Lambda \Lambda$ correlation function obtained by using the LL and KP formulae in comparison with data [14]. Right: Low-energy scattering parameters $\left(a_{0}, r_{\text {eff }}\right)$ of $\Lambda \Lambda$. Contours show $\chi^{2} / \mathrm{DOF}=0.65$ $\left(\lambda=(0.67)^{2}\right.$, solid contour) and $\chi^{2} / \mathrm{DOF}=0.56$ (free $\lambda$, dashed contour) in the LL model analysis of the correlation data. Symbols show $\left(1 / a_{0}, r_{\text {eff }}\right)$ from $\Lambda \Lambda$ potentials $[15,16,17,18]$, and shaded areas show the region favored by the $\Lambda \Lambda$ correlation data in Ref. [2](MFO2015). Filled black circle with $x y$ error bar shows the analysis result by the STAR collaboration, where $\lambda$ is regarded as a free parameter [14].

Recently, $\Lambda \Lambda$ correlation has been measured in high-energy heavy-ion collisions at the Relativistic Heavy-Ion Collider (RHIC) at the Brookhaven National Laboratory [14]. In order to access the $\Lambda \Lambda$ interaction, we also need to take account of the feed-down and residual-source effecs,

$$
C_{\text {corr }}(q)=\mathscr{N}\left[1+\lambda\left(C_{\text {bare }}(q)-1\right)+a_{\text {res }} e^{-4 r_{\text {res }}^{2} q^{2}}\right]
$$

where $C_{\text {bare }}$ is the bare correlation function (Eq. (2.2)), and $\mathscr{N}, \lambda, a_{\text {res }}$ and $r_{\text {res }}$ are the normalizaiton factor, pair purity, residual source strength and size, respectively.

In the left panel of Fig. 2, we compare the calculated correlation functions with data. We show the LL model results with $\lambda$ regarded as a free parameter (free $\lambda$ ) and with $\lambda=(0.67)^{2}$ (fixed $\lambda$ ), where the optimal scattering lengths are found to be positive and negative, respectively. In the free $\lambda$ case, the optimal $\lambda$ is found to be small $(\lambda \simeq 0.18$ ), then Eq. (3.1) with the quantum statistics and feed-down effects gives $C(q \rightarrow 0) \simeq 0.91$. In order to explain the data at small $q$ $\left(C_{\mathrm{STAR}}(0) \simeq 0.82\right)$, the $\Lambda \Lambda$ interaction and residual source need to suppress the correlation and a positive $a_{0}$ is favored. The above optimal $\lambda$ may be smaller than expected. In the experiment, $\Lambda \mathrm{s}$ from weak decays are excluded, then the main feed-down contribution should be the radiative decay of $\Sigma^{0}\left(\Sigma^{0} \rightarrow \Lambda \gamma\right)$. Then we can evaluate $\lambda \simeq\left[\Lambda /\left(\Lambda+\Sigma^{0}\right)\right]^{2} \simeq(0.67)^{2}$ using $\Sigma^{0} / \Lambda_{\text {tot }}=0.278$ in $\mathrm{p}+\mathrm{Be}$ reaction [19] and $\Xi / \Lambda_{\text {tot }}=0.15$ in heavy-ion collisions [20]. These ratios are roughly consistent with the statistical model estimate. By using the parameter set $\left(T, \mu_{B}, \mu_{s}\right)=(162,24,10) \mathrm{MeV}$ [1], 
one obtains $\Lambda /\left(\Lambda+\Sigma^{0}\right) \simeq 0.60(0.73)$ without (with) resonance decays of $\Sigma(1385), \Lambda(1405)$ and $\Lambda(1520)$. In the fixed $\lambda$ case, a negative $a_{0}$ is favored in order to enhance the correlation function at small $q$. The $\chi^{2} / \mathrm{DOF}$ in the fixed $\lambda$ case is larger than that in the free $\lambda$ case, but the flow effects reduce the correlation at small $q$ and lead to a better description of data.

Having these considerations in mind, it is reasonable to conclude that the scattering length is negative and there is no shallow bound state in $\Lambda \Lambda$. The constraint from the correlation, $1 / a_{0}<$ $-0.8 \mathrm{fm}^{-1}$ [2], is consistent with recently proposed $\Lambda \Lambda$ potentials $[16,17]$ and $\Lambda \Lambda$ potentials fitted to Nagara data [18]. The favored effective range is positive, then the pole around the $\Lambda \Lambda$ threshold would not be a resonance but a virtual pole. If this is the case, the resonance $H$ pole (if exists) should not be very close to the $\Lambda \Lambda$ threshold and would be a bound state or a resonance of $\Xi N$.

\section{2 $N \Omega$ interaction}

Another promising dibaryon candidate is $N \Omega$, where the color spin interaction is attractive [21] and the Pauli principle does not operate. Especially, the $J=2\left({ }^{5} \mathrm{~S}_{2}\right)$ state couples to the octet-octet baryon pair only with $L \geq 2$ partial waves, and the the width is expected to be small. Actually, the lattice QCD calculation [22] predicts a bound state. Thus it is interesting to make a prediction of the $\Omega^{-} p$ correlation [4].

In the upper panel of Fig. 3, we show the lattice QCD $N \Omega$ potential in the ${ }^{5} \mathrm{~S}_{2}$ channel at large quark masses $\left(m_{\pi}=895 \mathrm{MeV}\right)$ [22]. The data can be fitted by a function $V(r)=b_{1} e^{-b_{2} r^{2}}+b_{3}(1-$ $\left.e^{-b_{4} r^{2}}\right)\left(e^{-b_{5} r} / r\right)^{2}$, as shown by $V_{\mathrm{II}}$ in the figure. We also generate potentials, $V_{\mathrm{I}}$ and $V_{\mathrm{III}}$, by varying the range-parameter at long distance, $b_{5}$ [4]. The potentials $V_{\text {I,IIIIII }}$ have no bound state, one shallow bound state and a deep bound state, respectively, at physical baryon masses.

In the $\Omega^{-} p$ correlation, we need to take account of the Coulomb potential and ${ }^{3} \mathrm{~S}_{1}$ channel. The correlation function is given as

$$
C(q)=\int d \boldsymbol{r} S_{12}(\boldsymbol{r})\left[\left|\psi^{C}(\boldsymbol{r})\right|^{2}+\frac{5}{8}\left\{\left|\chi\left({ }^{5} S_{2}\right)\right|^{2}-\left|\psi_{0}^{C}(r)\right|^{2}\right\}+\frac{3}{8}\left\{\left|\chi\left({ }^{3} S_{1}\right)\right|^{2}-\left|\psi_{0}^{C}(r)\right|^{2}\right\}\right],
$$

where $\psi^{C}$ and $\psi_{0}^{C}$ are the Coulomb wave function and its $s$-wave component. The ${ }^{5} \mathrm{~S}_{2}$ wave function $\left(\chi\left({ }^{5} \mathrm{~S}_{2}\right)\right)$ is obtained with the strong $\left(V_{\mathrm{I}, \mathrm{II}, \mathrm{III}}\right)$ and Coulomb interactions. The coupling to the octet-octet channel in the $s$-wave leads to strong absorption in the ${ }^{3} \mathrm{~S}_{1}$ channel, we assume complete absorption for $r<r_{0}=2 \mathrm{fm}$ and only the Coulomb interaction for $r>r_{0}$ for $\chi\left({ }^{3} \mathrm{~S}_{1}\right)$.

In the lower left panel of Fig. 3, we show the $\Omega^{-} p$ correlation function without the Coulomb potential for $R=2.5 \mathrm{fm}$. The scattering lengths for $V_{\mathrm{I}, \mathrm{II}, \mathrm{III}}$ are $a_{0}=-1.0,23.1$ and $1.60 \mathrm{fm}$, which correspond to $R / a_{0}=-2.5,0.11$ and 1.56 , respectively. Thus the correlation functions show similar behaviors to those in Fig. 1.

When the Coulomb potential is switched on, the correlation function is strongly enhanced at small $q$ and the characteristic features from $R / a_{0}$ values are obscured. In order to shed light on the $N \Omega$ potential, we propose to introduce an "SL (small-to-large) ratio" of the correlation functions for different source sizes,

$$
C_{\mathrm{SL}}(q)=C_{R=2.5 \mathrm{fm}} / C_{R=5 \mathrm{fm}} .
$$

As shown in the lower right panel of Fig. $3, C_{\mathrm{SL}}$ shows behavior similar to that without the Coulomb interaction, since the Coulomb effect for small $q$ is largely cancelled. Thus the experimental obser- 


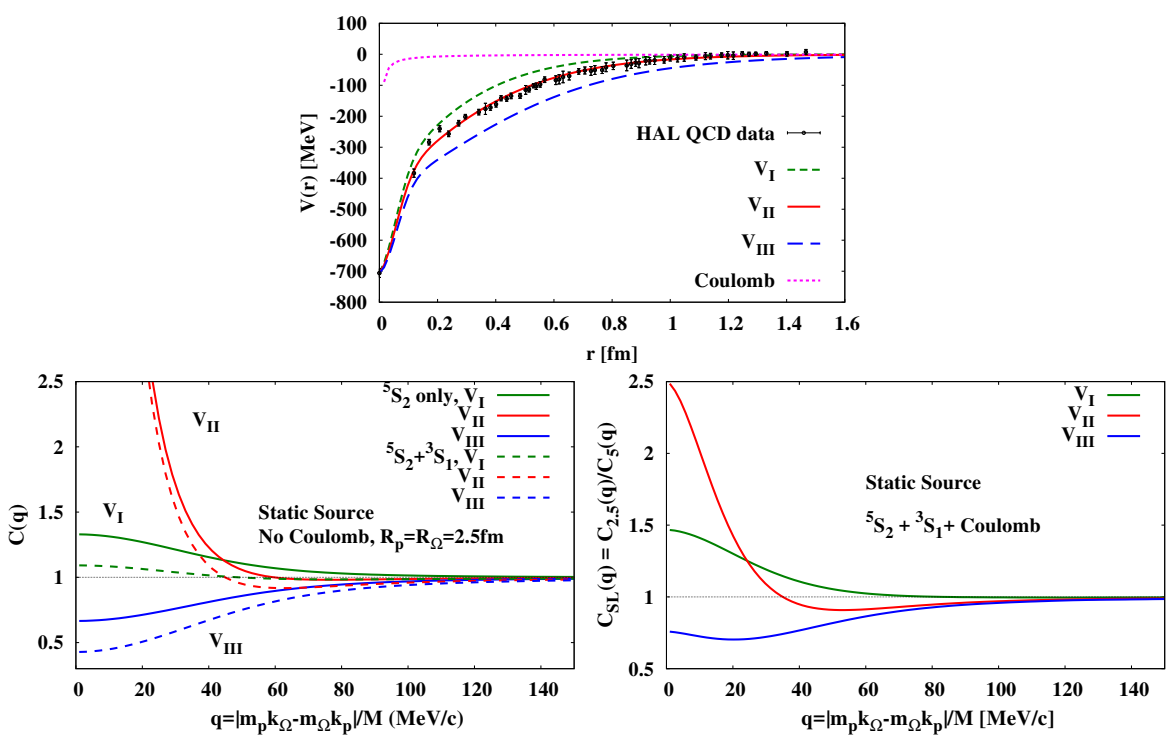

Figure 3: Upper panel: Three typical examples of the $N \Omega$ potential. The black circles show the lattice QCD data with heavy quark masses [22], and the solid line $\left(V_{\mathrm{II}}\right)$ is the fit to the lattice data. Dotted and dashed lines $\left(V_{\mathrm{I}, \mathrm{III}}\right)$ denote the potentials with weaker and stronger attractions, respectively. Lower left: $p \Omega$ correlation function for the static source with $R=2.5 \mathrm{fm}$ without the Coulomb interaction. Lower right: The small-large ratio $C_{\mathrm{SL}}(Q)$ for the static source between the different source sizes, $R=2.5$ and $5 \mathrm{fm}$.

vation of the SL ratio would be helpful to determine the sign and strength of the scattering length of the $N \Omega$ potential, and consequently to judge the existence of the $S=-3$ dibaryon state.

\section{3 $K^{-} p$ interaction}

The $\Lambda(1405)$ baryon resonance near the $\bar{K} N$ threshold is considered as a $\bar{K} N$ quasi-bound state [23]. The uncertainty of the $\bar{K} N$ scattering amplitude at around the threshold is reduced by the recent kaonic hydrogen data [24] combined with the $K^{-} p$ scattering data in the coupledchannel approach with chiral SU(3) dynamics [25]. Precise knowledge of the $\bar{K} N$ interaction is also important for the study of possible $\bar{K}$ bound states in nuclei [26]. Here we demonstrate that $K^{-} p$ correlation provides information on the interference of the $I=0$ and $I=1$ amplitudes.

In the $K^{-} p$ correlation, the coupled-channel effects with the $\bar{K}^{0} n$ channel play an important role. The outgoing wave function in the $K^{-} p$ channel is found to be [3],

$$
\psi_{K^{-} p}(r) \rightarrow \frac{1}{2 i q r}\left[e^{i q r}-\tilde{\mathscr{S}}_{K^{-} p}^{-1} e^{-i q r}\right], \quad \tilde{\mathscr{S}}_{K^{-} p}=2\left(\mathscr{S}_{0}^{-1}+\mathscr{S}_{1}^{-1}\right)^{-1}, \quad \mathscr{S}_{I}=e^{2 i \delta_{I}},
$$

where $\delta_{I}$ denotes the channel phase shift. It is interesting to find that $\tilde{\mathscr{S}}_{K^{-}}$is different from the $S$-matrix in the $K^{-} p$ scattering, $\mathscr{S}_{K^{-}}{ }=\left(\mathscr{S}_{0}+\mathscr{S}_{1}\right) / 2$.

In Fig. 4, we show the $K^{-} p$ correlation function obtained by using the KP and LL formulae with the $\bar{K} N$ potential in Ref. [27] based on the NLO chiral SU(3) dynamics [25]. The interaction range of the potential $(0.4 \mathrm{fm})$ is much smaller than the source size $R=3 \mathrm{fm}$, then both the KP and LL formulae predict similar results. It should be noted that the Coulomb interaction is not 


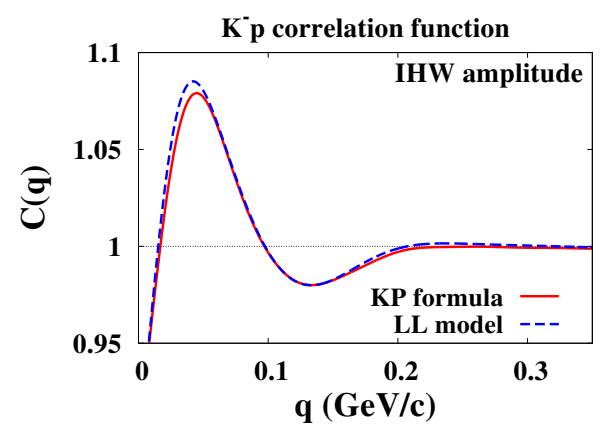

Figure 4: $K^{-} p$ correlation function obtained with the potential [27], which is fitted to the amplitudes [25]. Solid and dashed lines show the results in KP and LL formulae. The source size is set to be $R=3 \mathrm{fm}$.

included, and will modify the correlation function at small $q$. In the actual measurement, the $\Lambda(1520)$ resonance may affect the correlation around $q \sim 0.24 \mathrm{GeV} / c$.

It is interesting to note that the bump and dip structures around $q \sim 0.05 \mathrm{GeV} / c$ and $q \sim$ $0.12 \mathrm{GeV} / c$ do not appear in the $K^{-} p$ scattering at the corresponding energy. It turns out that these structures arise from the interference between two amplitudes of $I=0$ and $I=1$ components in $\tilde{S}_{K^{-}}$. In this way, the $K^{-} p$ correlation function gives information complementary to that from the elastic $K^{-} p$ scattering.

\section{Summary}

The two-particle momentum correlation in heavy-ion collisions shows characteristic dependence on the hadron-hadron interaction. For negative, large and positive scattering lengths, the correlation functions at small relative momenta are enhanced, strongly enhanced and suppressed, respectively, by the interaction. We have demonstrated that this characteristic behavior would be observed when the pair purity is sufficiently large. The scattering length of the $\Lambda \Lambda$ interaction is constrained to be $1 / a_{0}<-0.8 \mathrm{fm}^{-1}$ by the correlation data, if the pair purity is as large as the statistical model estimate. The "SL (small-to-large) ratio" of the $\Omega^{-} p$ correlation functions for different source sizes is found to show the above interaction dependence and to be useful to judge the existence of the $S=-3$ dibaryon state. The $K^{-} p$ correlation function is predicted to show non-monotonic behavior due to the interference of the $I=0$ and $I=1$ amplitudes. The correlation function is thus useful to obtain information on hadron-hadron interactions even for the pair of short-lived particles.

\section{Acknowledgement}

The author would like to thank the collaborators of Refs. [2, 3, 4], on which this proceedings is based, F. Etiminan, T. Furumoto, T. Hatsuda T. Hyodo, K. Miyahara and K. Morita. Numerical computations were carried out in part on SR16000 at YITP in Kyoto university. This work is supported in part by the Grants-in-Aid for Scientific Research from JSPS (Nos. 15K05079, 15H03663, and 16K05350) by the Grants-in-Aid for Scientific Research on Innovative Areas from MEXT (Nos. 24105001, 24105008). 


\section{References}

[1] S. Cho et al. [ExHIC Collaboration], Phys. Rev. Lett. 106 (2011) 212001; Phys. Rev. C 84 (2011) 064910; arXiv:1702.00486 [nucl-th].

[2] K. Morita, T. Furumoto and A. Ohnishi, Phys. Rev. C 91 (2015) 024916.

[3] A. Ohnishi, K. Morita, K. Miyahara and T. Hyodo, Nucl. Phys. A 954 (2016) 294.

[4] K. Morita, A. Ohnishi, F. Etminan and T. Hatsuda, Phys. Rev. C 94 (2016), 031901.

[5] S. E. Koonin, Phys. Lett. B 70 (1977) 43; S. Pratt et al., Phys. Rev. C 42 (1990) 2646; W. Bauer et al., Ann. Rev. Nucl. Part. Sci. 42 (1992) 77; M. A. Lisa et al., Ann. Rev. Nucl. Part. Sci. 55 (2005) 357.

[6] R. Lednicky and V. L. Lyuboshits, Sov. J. Nucl. Phys. 35 (1982) 770 [Yad. Fiz. 35 (1982) 1316].

[7] R. L. Jaffe, Phys. Rev. Lett. 38 (1977) 195 [Erratum-ibid. 38 (1977) 617].

[8] S. R. Beane et al. [NPLQCD Collaboration], Phys. Rev. Lett. 106 (2011) 162001; T. Inoue et al. [HAL QCD Collaboration], Phys. Rev. Lett. 106 (2011) 162002; J. Haidenbauer and U. G. Meissner, Phys. Lett. B 706 (2011) 100.

[9] H.Takahashi et al., Phys.Rev.Lett. 87 (2001) 212502; J.K.Ahn et al., Phys.Rev. C88 (2013), 014003.

[10] B. H. Kim et al. [Belle Collaboration], Phys. Rev. Lett. 110 (2013) 222002; J. Adam et al. [ALICE Collaboration], Phys. Lett. B 752 (2016) 267.

[11] T. Inoue et al. [HAL QCD Collaboration], Nucl. Phys. A 881 (2012) 28.

[12] C. J. Yoon et al. [KEK-PS E522 Collaboration], Phys. Rev. C 75 (2007) 022201(R).

[13] C. Greiner and B. Muller, Phys. Lett. B 219 (1989) 199;

A. Ohnishi, Y. Hirata, Y. Nara, S. Shinmura and Y. Akaishi, Nucl. Phys. A 670 (2000) 297.

[14] L. Adamczyk et al. [STAR Collaboration], Phys. Rev. Lett. 114 (2015) 022301.

[15] M. M. Nagels et al., Phys. Rev. D 15 (1977) 2547; Phys. Rev. D 20 (1979) 1633; P. M. M. Maessen et al., Phys. Rev. C 40 (1989) 2226; T. A. Rijken et al., Phys. Rev. C 59 (1999) 21; T. Ueda et al., Prog. Theor. Phys. 99 (1998) 891.

[16] T. A. Rijken, M. M. Nagels and Y. Yamamoto, Prog. Theor. Phys. Suppl. 185 (2010) 14.

[17] Y. Fujiwara, Y. Suzuki and C. Nakamoto, Prog. Part. Nucl. Phys. 58 (2007) 439.

[18] I. N. Filikhin and A. Gal, Nucl. Phys. A 707 (2002) 491;

E. Hiyama et al., Phys. Rev. Lett. 104 (2010) 212502; Phys. Rev. C 66 (2002) 024007.

[19] M. W. Sullivan et al., Phys. Rev. D 36 (1987) 674.

[20] G. Agakishiev et al. [STAR Collaboration], Phys. Rev. Lett. 108 (2012) 072301.

[21] M. Oka, Phys. Rev. D38 (1988) 298; A. Gal, Acta Phys. Polon. B47 (2016) 471.

[22] F. Etminan et al. [HAL QCD Collaboration], Nucl. Phys. A928 (2014) 89.

[23] R. H. Dalitz and S. F. Tuan, Annals Phys. 10 (1960) 307; N. Kaiser, P. B. Siegel and W. Weise, Nucl. Phys. A 594 (1995) 325; T. Hyodo and D. Jido, Prog. Part. Nucl. Phys. 67 (2012) 55.

[24] M. Bazzi et al. [SIDDHARTA Collaboration], Phys. Lett. B 704 (2011) 113.

[25] Y. Ikeda, T. Hyodo and W. Weise, Phys. Lett. B 706 (2011) 63; Nucl. Phys. A 881 (2012) 98.

[26] Y. Nogami, Phys. Lett. 7 (1963) 288; Y. Akaishi and T. Yamazaki, Phys. Rev. C 65 (2002) 044005;

D. Gazda and J. Mares, Nucl. Phys. A 881 (2012) 159.

[27] K. Miyahara and T. Hyodo, Phys. Rev. C 93 (2016) 015201. 